Sitzungsber. Abt. II (2005) 214: 101-109 Sitzungsberichte

Mathematisch-naturwissenschaftliche Klasse Abt. II

Mathematische, Physikalische und Technische Wissenschaften

(๖) Österreichische Akademie der Wissenschaften 2006

Printed in Austria

\title{
Erweiterung eines ebenen Kollinearitätsproblems
}

\author{
Von \\ Oswald Giering \\ (Vorgelegt in der Sitzung der math.-nat. Klasse am 13. Oktober 2005 \\ durch das k. M. Oswald Giering)
}

\section{Problemstellung und Ergebnisse}

Kollinearitätsaussagen wie der Satz von Pascal über die PascalGeraden [1, S. 208], der Satz von Wallace über die Wallace-Geraden [1, S. 154 f.] und der Satz von Euler über die Euler-Gerade eines Dreiecks [1, S. 148] zählen zum klassischen Bestand der Elementargeometrie. Eine weitere Kollinearitätsaussage ergab sich in [2] als Lösung des folgenden Kollinearitätsproblems:

In der reellen projektiven Ebene seien $P_{0}, P_{1}, P_{2}$ drei linear unabhängige Punkte (Grundpunkte) und $g_{0}, g_{1}, g_{2}$ drei paarweise verschiedene Geraden (Leitgeraden). Die Projektionen der Grundpunkte $P_{i}(i=0,1,2)$ aus einem laufenden Punkt $P$ auf die Leitgeraden $g_{i}$ seien die Punkte $S_{i}:=g_{i} \cap P P_{i}$. Gesucht sind alle Punkte $P$ mit kollinearen Schnittpunkten $S_{i}$. Als Ort der Punkte $P$ stellte sich in [2] eine Kubik (Kurve 3. Ordnung) ein.

Wir studieren nun eine Erweiterung dieses Kollinearitätsproblems (Abb. 1): Wir ersetzen die Leitgeraden $g_{i}$ durch drei paarweise verschiedene, nichtentartete Kegelschnitte (Leitkegelschnitte) $k_{i}$, betrachten zu einem laufenden Punkt $P$ erneut die drei Geraden $P P_{i}$ und sodann jeweils ihren Pol $\left(k_{i}-P o l\right) S_{i}(i=0,1,2)$ bezüglich des Leitkegelschnitts $k_{i}$. Gesucht seien alle Punkte $P$ mit kollinearen $k_{i^{-}}$ Polen $S_{i}$. Ist die Kollinearitätsbedingung nicht identisch erfüllt, dann 


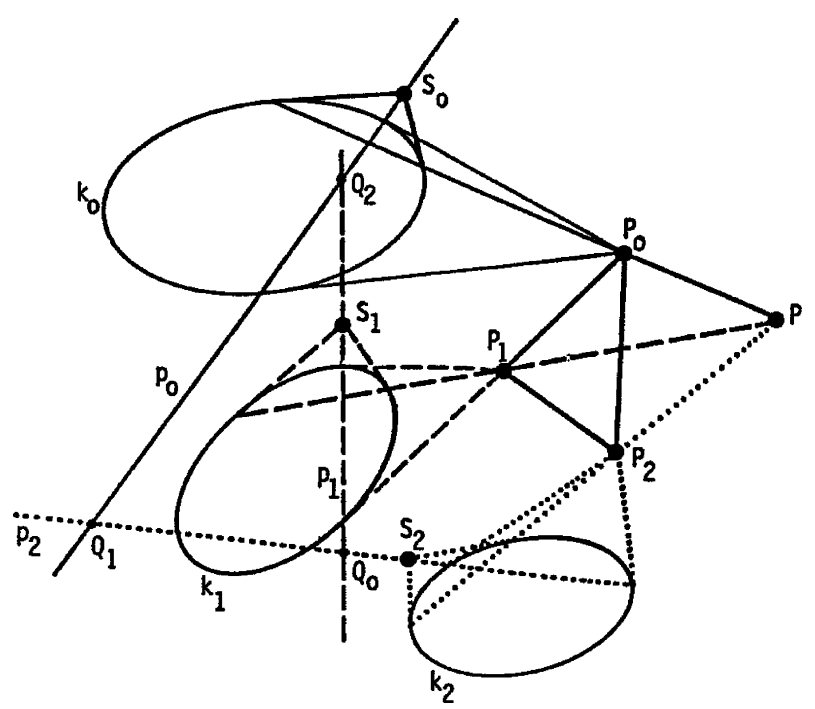

Abb. 1

stellt sich als Ort der Punkte $P$ erneut eine (die Grundpunkte $P_{i}$ durchlaufende) Kubik $k$ ein. Unser weiteres Interesse gilt möglichst elementaren Kollinearitätsaussagen, die unter das erweiterte Kollinearitätsproblem fallen. Wir geben dafür drei Beispiele, in denen wir als Leitkegelschnitte Kreise wählen. Im ersten Beispiel sind die Seiten des Grundpunktedreiecks $P_{0} P_{1} P_{2}$ Durchmesser der Leitkreise. Die aus drei Ästen bestehende Kubik $k$ durchläuft dann die Ecken, den Schwerpunkt und den Höhenschnittpunkt des Dreiecks $P_{0} P_{1} P_{2}$ (Abb. 2). Im zweiten und dritten Beispiel werden die Grundpunkte $P_{i}$ und Leitkreise $k_{i}$ derart gewählt, dass die Kubik $k$ zerfällt (Abb. 3 und 5).

\section{Projektive Behandlung des Problems}

Die linear unabhängigen Grundpunkte $P_{i}(i=0,1,2)$ seien die Grundpunkte eines projektiven $\left(x_{0}, x_{1}, x_{2}\right)$-Koordinatensystems; ihre Koordinaten seien

$$
P_{0}=(1,0,0), \quad P_{1}=(0,1,0), \quad P_{2}=(0,0,1) .
$$

Die Gleichungen der Leitkegelschnitte $k_{i}$ seien

$$
\begin{aligned}
k_{0}: & a x_{0}^{2}+b x_{1}^{2}+c x_{2}^{2}+2 d x_{0} x_{1}+2 e x_{1} x_{2}+2 f x_{2} x_{0}=0, \\
k_{1}: & \alpha x_{0}^{2}+\beta x_{1}^{2}+\gamma x_{2}^{2}+2 \delta x_{0} x_{1}+2 \varepsilon x_{1} x_{2}+2 \varphi x_{2} x_{0}=0, \\
k_{2}: & A x_{0}^{2}+B x_{1}^{2}+C x_{2}^{2}+2 D x_{0} x_{1}+2 E x_{1} x_{2}+2 F x_{2} x_{0}=0 .
\end{aligned}
$$


Da jeder Leitkegelschnitt $k_{i}$ fünf wesentliche Koeffizienten besitzt, hängt das erweiterte Kollinearitätsproblem nach (2) von 15 wesentlichen Parametern $\mathrm{ab}$. Berechnet man $\mathrm{zu}$ einem laufenden Punkt $P=\left(\xi_{0}, \xi_{1}, \xi_{2}\right)$ die Koordinaten $\left(s_{00}, s_{01}, s_{02}\right)$ des $k_{0}$-Pols $S_{0}$ der Geraden $P P_{0}$, so erhält man

$$
\begin{aligned}
& d_{0} s_{00}=(b f-d e) \xi_{1}+(e f-c d) \xi_{2}, \\
& d_{0} s_{01}=(a e-d f) \xi_{1}+\left(a c-f^{2}\right) \xi_{2}, \\
& d_{0} s_{02}=\left(d^{2}-a b\right) \xi_{1}+(a e-d f) \xi_{2}
\end{aligned}
$$

mit

$$
d_{0}:=\left|\begin{array}{lll}
a & d & f \\
d & b & e \\
f & e & c
\end{array}\right| \neq 0 .{ }^{1}
$$

Für die Koordinaten $\left(s_{10}, s_{11}, s_{12}\right)$ des $k_{1}$-Pols $S_{1}$ und die Koordinaten $\left(s_{20}, s_{21}, s_{22}\right)$ des $k_{2}$-Pols $S_{2}$ findet man aus (2) analoge Linearformen in den Koordinaten $\xi_{i}$. Die $k_{i}$-Pole $S_{i}(i=0,1,2)$ sind genau dann kollinear, wenn gilt:

$$
\begin{aligned}
& \operatorname{det}\left(s_{i 0} \quad s_{i 1} \quad s_{i 2}\right) \\
& =\left|\begin{array}{cc}
(b f-d e) \xi_{1}+(e f-c d) \xi_{2} & (a e-d f) \xi_{1}+\left(a c-f^{2}\right) \xi_{2} \\
(\delta \varepsilon-\beta \varphi) \xi_{0}+\left(\varepsilon^{2}-\beta \gamma\right) \xi_{2} & (\delta \varphi-\alpha \varepsilon) \xi_{0}+(\gamma \delta-\varepsilon \varphi) \xi_{2} \\
(C D-E F) \xi_{0}+\left(B C-E^{2}\right) \xi_{1} & \left(F^{2}-A C\right) \xi_{0}-(C D-E F) \xi_{1} \\
& \left(d^{2}-a b\right) \xi_{1}-(a e-d f) \xi_{2} \\
& \left(\alpha \beta-\delta^{2}\right) \xi_{0}-(\delta \varepsilon-\beta \varphi) \xi_{2} \\
(A E-D F) \xi_{0}-(D E-B F) \xi_{1}
\end{array}\right| \\
& =0 .
\end{aligned}
$$

Man entnimmt aus (4) den folgenden Satz 1.

Satz 1 (Abb. 1). Die $k_{i}$-Pole $S_{i}$ der Geraden $P P_{i}$ bezüglich des Kegelschnitts $k_{i}(i=0,1,2)$ sind - wenn (4) nicht identisch erfüllt ist genau dann kollinear, wenn P ein Punkt der durch (4) gegebenen Kubik $k$ ist. Die Kubik $k$ durchläuft die Grundpunkte $P_{0}, P_{1}, P_{2}$. Die Kubik $k$ kann zerfallen und wird auch als Kollinearitätskubik bezeichnet.

Bemerkungen: (1) Der $k_{i}$-Pol $S_{i}$ der Geraden $P P_{i}$ durchläuft die $k_{i}$-Polare $p_{i}$ des Grundpunkts $P_{i}$, wenn $P$ die Punkte der Ebene

\footnotetext{
${ }^{1}$ Mit $d_{0} \neq 0$ ist $k_{0}$ ein nicht zerfallender (nicht entarteter) Kegelschnitt.
} 
durchläuft. Die drei $k_{i}$-Polaren können zusammenfallen. In diesem Fall sind die $k_{i}$-Pole $S_{i}$ für alle Punkte $P$ der Ebene kollinear; (4) stellt dann keine Bedingung dar.

Wenn zwei der drei $k_{i}$-Polaren $p_{i}$ zusammenfallen (etwa $p_{1}=p_{2}$ ), dann liegen kollineare $k_{i}$-Pole $S_{0}, S_{1}, S_{2}$ stets auf $p_{1}=p_{2}$, und es ist $S_{0}=p_{0} \cap p_{1}=p_{0} \cap p_{2}$. Die Punkte $P$ mit kollinearen $k_{i}$-Polen $S_{0}, S_{1}, S_{2}$ sind dann die Punkte der Geraden $P P_{0}$ mit dem $k_{0}$-Pol $S_{0}=p_{0} \cap p_{1}=p_{0} \cap p_{2}$.

Im allgemeinen Fall bilden die $k_{i}$-Polaren $p_{i}(i=0,1,2)$ ein Dreiseit $p_{0} p_{1} p_{2}$ mit den Ecken (Abb. 1)

$$
Q_{0}:=p_{1} \cap p_{2}, \quad Q_{1}:=p_{2} \cap p_{0}, \quad Q_{2}:=p_{0} \cap p_{1} .
$$

Das Dreiseit $p_{0} p_{1} p_{2}$ kann in drei (paarweise verschiedene) kopunktale Geraden entarten.

(2) Nach Satz 1 liegen die Grundpunkte $P_{0}, P_{1}, P_{2}$ auf der Kollinearitätskubik $k$. Bilden die $k_{i}$-Polaren $p_{i}$ ein nicht entartetes Dreiseit mit den Ecken $Q_{0}, Q_{1}, Q_{2}$, dann findet man wie folgt drei weitere ausgezeichnete Punkte der Kubik $k$ : Man fixiere zunächst jene Gerade $h_{0}$ durch $P_{0}$, deren $k_{0}$-Pol $S_{0}=Q_{2}$ ist. Ebenso fixiere man jene Gerade $h_{1}$ durch $P_{1}$, deren $k_{1}$-Pol $S_{1}=Q_{2}$ ist. Dann ist für $h_{0} \neq h_{1}$ der Schnittpunkt $h_{0} \cap h_{1}$ ein Punkt der Kubik $k$ (denn wegen $S_{0}=S_{1}=Q_{2}$ sind $S_{0}, S_{1}, S_{2}$ kollinear). Für $h_{0}=h_{1}$ liegt jeder Punkt der Geraden $h_{0}=h_{1}$ auf $k ; k$ zerfällt in $h_{0}=h_{1}$ und einen Kegelschnitt. Zwei weitere ausgezeichnete Punkte der Kubik $k$ findet man bezüglich $Q_{0}$ und $Q_{1}$.

(3) Die Grundpunkte $P_{0}, P_{1}, P_{2}$ wurden bisher linear unabhängig vorausgesetzt. Sind $P_{0}, P_{1}, P_{2}$ linear abhängig und paarweise verschieden, dann lässt sich das erweiterte Kollinearitätsproblem in entsprechender Weise projektiv behandeln.

\section{Beispiele}

Wir diskutieren in diesem Abschnitt drei Beispiele des erweiterten Kollinearitätsproblems, die ein eigenes Interesse verdienen. Als Leitkegelschnitte wählen wir jeweils Kreise. Die auftretenden Kollinearitätskubiken lassen sich in einem (jedem Beispiel angepassten) kartesischen Koordinatensystem bequem berechnen.

\subsection{Beispiel 1}

Abb. 2 visualisiert den folgenden Satz 2.

Satz 2. Die Grundpunkte $P_{0}, P_{1}, P_{2}$ seien die Ecken eines nicht rechtwinkligen Dreiecks. Die Leitkreise $k_{0}, k_{1}, k_{2}$ seien die Kreise 


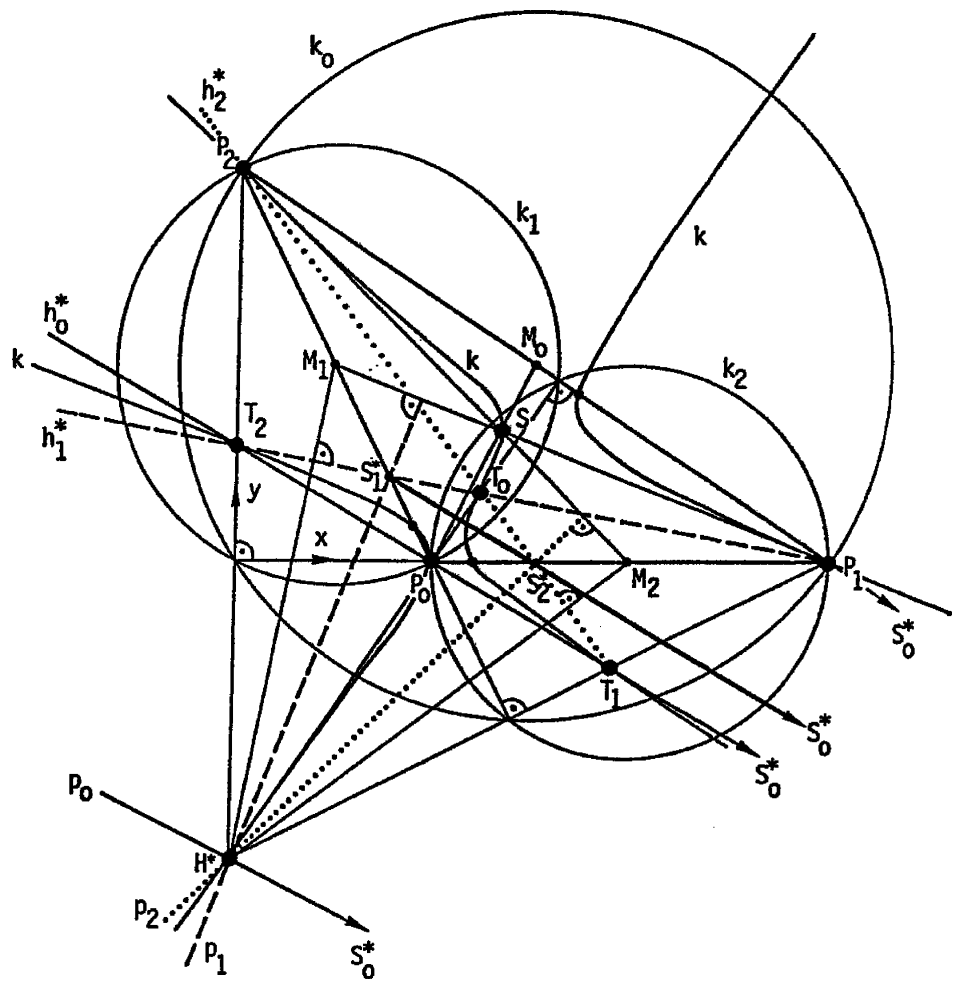

Abb. 2

über den Seiten des Grundpunktedreiecks; $k_{i}(i=0,1,2)$ sei der Kreis, der nicht durch $P_{i}$ geht. Dann geht die (nicht zerfallende) Kollinearitätskubik $k$ durch

(1) die Grundpunkte $P_{0}, P_{1}, P_{2}$,

(2) den Schwerpunkt $S$ des Dreiecks $P_{0} P_{1} P_{2}$,

(3) den Höhenschnittpunkt $H^{*}$ des Dreiecks $P_{0} P_{1} P_{2}$,

(4) die Ecken $T_{0}, T_{1}, T_{2}$ des Dreiseits $h_{0}^{*} h_{1}^{*} h_{2}^{*}$ aus den $k_{i}$-Polaren $h_{i}^{*}(i=0,1,2)$ von $H^{*}$.

Außerdem gilt:

(5) Die Ecke $T_{i}$ liegt auf der Höhe $H^{*} P_{i}$ des Dreiecks $P_{0} P_{1} P_{2}$.

(6) Die Kollinearitätskubik $k$ berührt die Seitenhalbierende $P_{i} M_{i}$ des Dreiecks $P_{0} P_{1} P_{2}$ in $P_{i} ; M_{i}$ ist die Mitte der Gegenseite von $P_{i}$.

(7) Die $k_{i}$-Polaren $p_{i}$ (siehe Abb. 1 und 2) sind kopunktal in $H^{*}$. 
Beweis. Zu (1): Nach Satz 1 durchläuft die Kollinearitätskubik $k$ die Grundpunkte $P_{0}, P_{1}, P_{2}$.

$\mathrm{Zu}$ (2): Der $k_{i}$-Pol der Seitenhalbierenden $S P_{i}(i=0,1,2)$ des Dreiecks $P_{0} P_{1} P_{2}$ ist ein Fernpunkt $F_{i}$. Die drei Fernpunkte $F_{i}$ sind kollinear auf der Ferngeraden, folglich ist $S$ ein Punkt der Kubik $k$.

$\mathrm{Zu}$ (3): Gibt man den Grundpunkten $P_{i}$ im $(x, y)$-Koordinatensystem der Abb. 2 die Koordinaten

$$
P_{0}=(a, 0), \quad P_{1}=(b, 0), \quad P_{2}=(0, c),
$$

so folgt $H^{*}=(0,-a b / c)$, und als Gleichung der Kollinearitätskubik $k$ berechnet man

$$
\begin{aligned}
& \left|\begin{array}{ccc}
b c(a-x)-\left(c^{2}+2 a b\right) y & b^{2}(a-x)+(2 a-b) c y & c(a-x)-(2 a-b) y \\
a c(b-x)-\left(c^{2}+2 a b\right) y & a^{2}(b-x)+(2 b-a) c y & c(b-x)-(2 b-a) y \\
4 a b(c-y)-2 c(a+b) x & (a-b)^{2} x & (a+b)(c-y)-2 c x
\end{array}\right| \\
& \quad=0 .
\end{aligned}
$$

Die Koordinaten von $H^{*}$ erfüllen (7).

$\mathrm{Zu}$ (4): Der Punkt $T_{0}=h_{1}^{*} \cap h_{2}^{*}$ ist ein Punkt der Kubik $k$, wenn die drei $k_{i}$-Pole der Geraden $T_{0} P_{i}(i=0,1,2)$ kollinear sind. Wegen $T_{0}=h_{1}^{*} \cap h_{2}^{*}$ ist $H^{*}$ der $k_{1}$-Pol von $T_{0} P_{1}$ und zugleich der $k_{2}$-Pol von $T_{0} P_{2}$. Damit sind die drei $k_{i}$-Pole der Geraden $T_{0} P_{i}$ kollinear. Ebenso sind $T_{1}=h_{2}^{*} \cap h_{0}^{*}$ und $T_{2}=h_{0}^{*} \cap h_{1}^{*}$ Punkte der Kubik $k$.

$\mathrm{Zu}$ (5): Berechnet man die Koordinaten von $T_{2}$, so folgt

$$
T_{2}=\left(0, \frac{a b c}{c^{2}+2 a b}\right) \in H^{*} P_{2} .
$$

Die zulässige zyklische Vertauschung $0 \rightarrow 1 \rightarrow 2 \rightarrow 0$ liefert $T_{0} \in H^{*} P_{0}, T_{1} \in H^{*} P_{1}$.

$\mathrm{Zu}$ (6): Diese Eigenschaft ergibt sich mit Hilfe von (7).

$\mathrm{Zu}$ (7): Eine einfache Rechnung zeigt: Die Koordinaten von $H^{*}$ erfüllen die Gleichung jeder $k_{i}$-Polaren $p_{i}(i=0,1,2)$.

In Abb. 2 schneiden je zwei Leitkreise einander in einer Ecke und dem zugehörigen Höhenfußpunkt des Grundpunktedreiecks. Die $k_{i}$-Polare $h_{i}^{*}$ von $H^{*}$ und die Gerade $H^{*} M_{i}$ sind in Abb. 2 (nach einer elementaren Eigenschaft der Polarentheorie der Kreise) orthogonal. Abb. 2 zeigt außerdem die wegen $H^{*} \in k$ kollinearen $k_{i}$-Pole $S_{i}^{*}$ der Geraden $H^{*} P_{i}(i=0,1,2)$. Nach Satz 2 kennt man mit $H^{*}, P_{i}, T_{i}(i=0,1,2)$ die Schnittpunkte der Kollinearitätskubik $k$ mit jeder Höhe des Grundpunktedreiecks; die Schnittpunkte von $k$ mit den Seitenhalbierenden sind $S_{i}$ und $P_{i}\left(P_{i}\right.$ nach Satz 2, Aussage (6) zweifach zählend). 


\subsection{Beispiel 2}

Abb. 3 zeigt drei paarweise verschiedene Leitkreise $k_{i}(i=0,1,2)$, eine jeden Leitkreis schneidende Gerade $t$ mit kollinearen $k_{i}$-Polen $T_{i}$ sowie drei paarweise verschiedene Grundpunkte $P_{i} \in t ; P_{i}$ liegt im Mittelpunkt der von $t$ aus $k_{i}$ ausgeschnittenen Sehne. Für jeden Punkt $P \in t$ ist $T_{i}$ der $k_{i}$-Pol der Geraden $P P_{i}=t$. Die Gerade $t$ ist daher ein Teil der Kollinearitätskubik $k$. Insgesamt zerfällt $k$ in die Gerade $t$ und ein $P_{i} T_{i}$-paralleles (reelles, konjugiert komplexes oder zusammenfallendes) Geradenpaar $l_{1}, l_{2}$. Für jeden Punkt $P \in l_{1} \cup l_{2}$ sind die $k_{i}$-Pole $S_{i}$ der Geraden $P P_{i}$ kollinear.

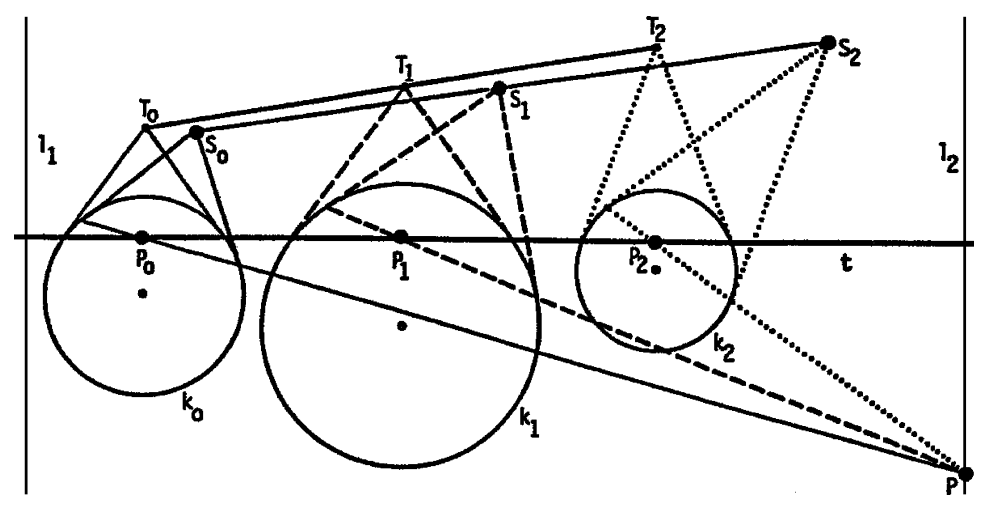

Abb. 3

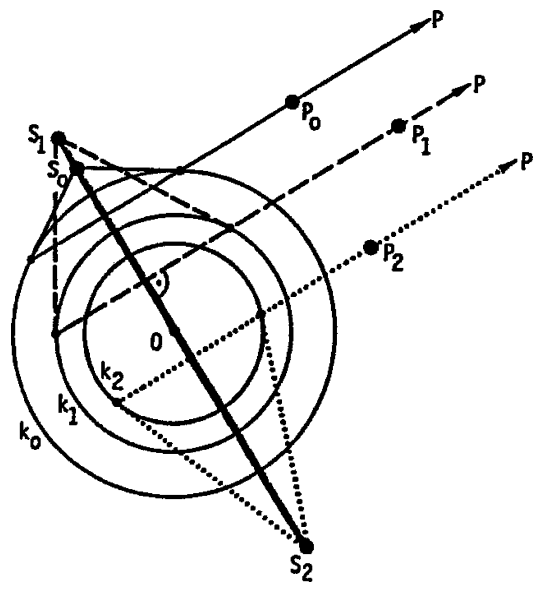

Abb. 4 


\subsection{Beispiel 3}

Abb. 4 zeigt drei konzentrische Leitkreise $k_{i}$ mit dem Mittelpunkt $O$ und drei linear unabhängige Grundpunkte $P_{i}(i=0,1,2)$. In diesem Fall sind die Geraden $P P_{i}$ für jeden Fernpunkt $P$ parallel. Ihre $k_{i}$-Pole $S_{i}$ sind folglich kollinear und zugleich kollinear mit $O$; die Geraden $P P_{i}$ und $O S_{i}$ sind stets orthogonal. Die Kollinearitätskubik $k$ zerfällt daher in die Ferngerade und einen Kegelschnitt $k^{\prime}$ (der den Mittelpunkt $O$ und die Grundpunkte $P_{i}$ enthält). ${ }^{2}$

Bei geeigneter gegenseitiger Lage der Leitkreise $k_{i}$ und der Grundpunkte $P_{i}$ stellt sich als Kegelschnitt $k^{\prime}$ ein Kreis ein. Man findet dann unschwer den folgenden Satz 3 (Abb. 5), der sich unabhängig von dem bisher untersuchten Kollinearitätsproblem formulieren lässt:

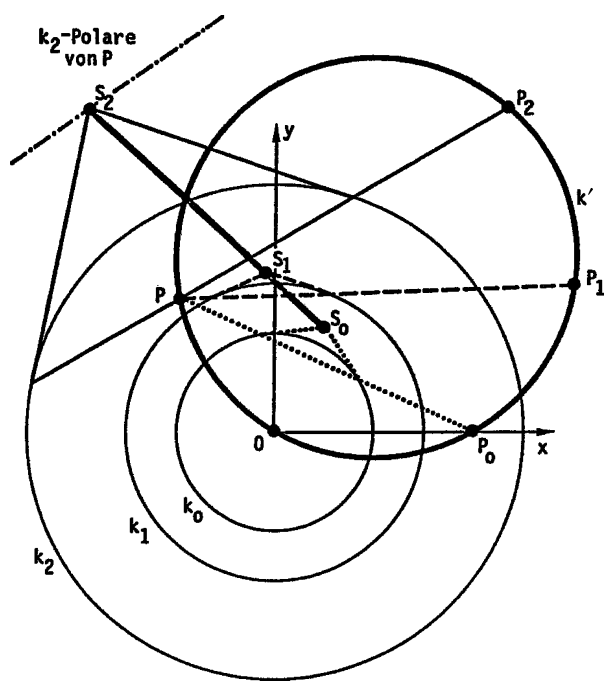

Abb. 5

Satz 3. Seien $k_{i}(i=0,1,2)$ konzentrische Kreise und $k^{\prime}$ ein Kreis durch ihren Mittelpunkt $O$. Auf $k^{\prime}$ seien $P_{0}$ und $P_{1}$ feste Punkte, $P$ sei ein laufender Punkt auf $k^{\prime}$. Dann gibt es auf $k^{\prime}$ einen eindeutig bestimmten Punkt $P_{2}$, so dass für alle Punkte $P \in k^{\prime}$ die $k_{i}$-Pole $S_{i}$ der Kreissehnen $P P_{i}$ kollinear sind.

\footnotetext{
${ }^{2}$ Sind zwei Grundpunkte $P_{i}$ - etwa $P_{0}$ und $P_{1}$ - kollinear mit $O$, dann zerfällt $k^{\prime}$ in die Gerade $P_{0} P_{1}$ und eine Gerade durch $P_{2}$.
} 
Ausgehend von einem beliebig gewählten Punkt $P \in k^{\prime}$ läßt sich der Punkt $P_{2} \in k^{\prime}$ in drei Schritten konstruieren (Abb. 5). Man bestimme (1) zu $P P_{0}$ den $k_{0}$-Pol $S_{0}$ und zu $P P_{1}$ den $k_{1}$-Pol $S_{1}$, (2) den Schnittpunkt $\left(=S_{2}\right)$ der Geraden $S_{0} S_{1}$ mit der $k_{2}$-Polaren von $P$, (3) die $k_{2}$-Polare von $S_{2}$; ihre Schnittpunkte mit $k^{\prime}$ sind $P$ und $P_{2}$.

\section{Literatur}

[1] Koecher, M., Krieg, A. (2000) Ebene Geometrie, 2. Aufl. Springer, Berlin Heidelberg New York

[2] GIERING, O. (2005) Ein ebenes Kollinearitätsproblem. IBDG 24(1): 23-32

Anschrift des Verfassers: Prof. Dr. Oswald Giering, Zentrum Mathematik, TU München, 85748 Garching, Deutschland. 